\title{
Methemoglobinemia, CTCAE
}

National Cancer Institute

\section{Source}

National Cancer Institute. Methemoglobinemia, CT CAE. NCI Thesaurus. Code C143191.

A disorder characterized by laboratory test results that indicate increased

methemoglobin in the blood. 